\title{
Schnellerer Wirkeintritt - weniger intra- und postoperative Schmerzen
}

Das Enzym Hyaluronidase ist ein bewährtes Adjuvans zur Unterstützung der Lokalanästhesie im ophtalmologischen Bereich, insbesondere im Rahmen der Retro- und Peribulbäranästhesie. Als Zusatz zur Infiltrationsanästhesie ist es auch bei chirurgischen Eingriffen an den Augenlidern hilfreich.

„Wir wollen möglichst wenig Lokalanästhesie bei Augenoperationen, dabei aber ein gutes Eindringen und viel Effekt, daher brauchen wir die Hyaluronidase“, erklärte Tip Dr. Ägäis Univ. H. Aral, niedergelassener Augenarzt mit Praxisklinik in Köln. Die Wirksamkeit des Enzyms Hyaluronidase (Hylase ${ }^{\circledast}$ Dessau) beruht auf seinem „Spreading-Effekt“: Hyaluronidase spaltet komplexe Kohlenhydrate in der extrazellulären Matrix. Die Konse- quenz daraus ist, dass sich der freie Wasseranteil im Gewebe erhöht, die Viskosität im Gewebe nimmt ab. Durch diese Prozesse wird die Durchlässigkeit des Bindegewebes gesteigert, was eine Diffusionsbeschleunigung des eingesetzten Lokalanästhetikums bewirkt. Durch das bessere Eindringen des Betäubungsmittels kommt es zu einem schnelleren Wirkeintritt und einem größeren schmerzunempfindlichen Bereich. Zusätzlich entstehen weniger Schwellungen, Hämatome und intra- und postoperative Schmerzen.

Nicht nur bei der Retro- und Peribulbäranästhesie, z. B. im Rahmen der Kataraktchirurgie, sondern auch bei der Blepharoplastik ist der Zusatz von Hyaluronidase wichtig. Chirurgische Eingriffe am Augenlid sind ein Grenzgebiet zwischen funktioneller Störung und kosmetisch-ästhetischer Chirurgie. Denn nicht nur ästhetische Gründe sind eine Indikation für die Operation, sondern manche Befunde sind so stark, dass es zu einer Gesichtsfeldeinschränkung kommt. Auch hier ist nach Erfahrung von Prof. Karl-Heinz Emmerich, Augenarzt in Darmstadt, die Zugabe von $0,5 \mathrm{ml}$ Hyaluronidase zur Infiltrationsanästhesie sinnvoll. „In diesem Bereich muss die Anästhesie sitzen, sonst können Sie nicht operieren“, erklärte Prof. Emmerich. Aufgrund der geringeren Blutungsgefahr bevorzugt er die Korrektur mithilfe eines $\mathrm{CO}_{2}$-Lasers. Durch den Einsatz von Hyaluronidase sei dies trotz der thermischen Reizung in Lokalanästhesie möglich und alle lidchirurgischen Maßnahmen können schmerzfrei durchgeführt werden.

Susanne Kammerer

Pressekonferenz "Fokus Gesicht: OphthalmoChirurgie trifft Plastische Chirurgie - Einsatz von Hylase ${ }^{\circledast}$ Dessau“, Berlin, 2.10.2015; Veranstalter: RIEMSER Pharma GmbH, Greifswald

\section{Postoperative Narbenpflege kann viel zur Prophylaxe beitragen}

30-50\% der Patienten sind mit ihren Narben nach Trauma oder Operation unzufrieden, was eine erhebliche psychische Belastung darstellen kann. Dies gilt insbesondere für deutlich über dem Hautniveau liegende, verhärtete und gerötete unphysiologische Narben, die bei

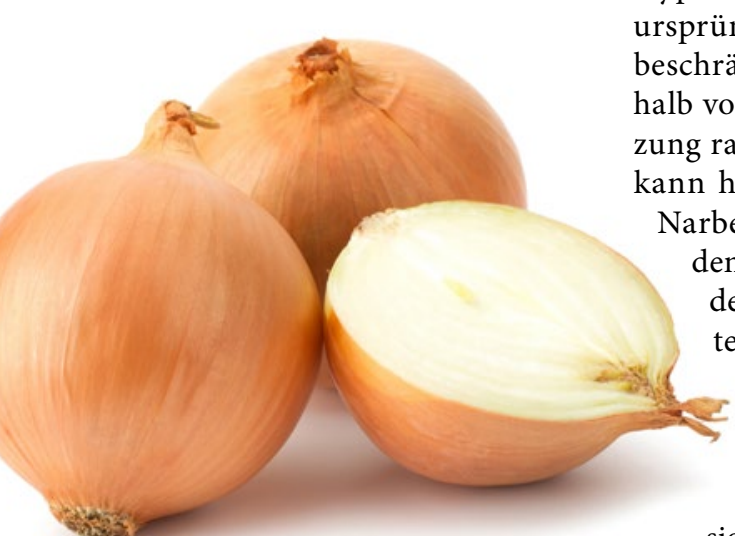

Ein Narbengel mit Zwiebelextrakt kann zur Vermeidung unschöner Narben beitragen. ungünstiger Lage auch zu Bewegungseinschränkungen führen können.

Bei der unphysiologischen Narbenbildung muss man zwischen hypertrophen Narben und Narbenkeloid unterscheiden, sagte Dr. Uwe Schwichtenberg, niedergelassener Dermatologe aus Bremen. Hypertrophe Narben bleiben auf den ursprünglichen Verletzungsbereich beschränkt, vergrößern sich aber innerhalb von 2-6 Monaten nach der Verletung rasch. Die endgültige Ausreifung ann hier bis zu 2 Jahre dauern. Ein

Narbenkeloid überschreitet dagegen den Rand der ursprünglichen Wunde und kann mehrere Jahre weiterwachsen.

Eine geeignete Narbenpflege kann bei der Vermeidung unschöner Narben helfen. Neben Narbenmassage und Kompression hat sich hier ein Narbengel mit einer Kombination der Wirkstoffe Heparin, Extractum cepae (Zwiebelextrakt) und Allantoin bewährt, das in den Leitlinien sowohl zur Zusatztherapie hypertropher aktiver Narben als auch zur postoperativen Prophylaxe empfohlen wird. Belegt ist der positive Effekt unter anderem in einer Studie von Willital et al, in der bei frühzeitiger Anwendung des Narbengels eine effektive Reduktion von Spannung, Schmerzen, Juckreiz, Rötung und Verhärtung belegt werden konnte (Willital GH et al. J Drugs Dermatol 2013;12:38-42).

Als Neuentwicklung stellte der Dermatologe hier ein Patch für die Nacht vor (Contractubex ${ }^{\circledast}$ Intensivpatch für die Nacht), das für die Patienten auch an schwer zu erreichenden Körperstellen besonders leicht in der Anwendung ist. $\mathrm{Zu}$ sätzlich zu der gleichmäßigen Freisetzung der Wirkstoffe hat man hier einen okklusiven Effekt, wodurch ein günstiges feuchtes Klima geschaffen und damit das Eindringen der Wirkstoffe erleichtert wird.

Maria Weiß

Vortrag „Die optimale Narbe von der Schnittführung bis zur Narbenbehandlung" bei der 13. Jahrestagung der Berliner Dermatologen dermapraxis Berlin“, Berlin, 18.9.2015; Veranstalter: Merz

Pharmaceuticals 